Proyecciones

Vol. 18, No 2, pp. 183-193, December 1999

Universidad Católica del Norte

Antofagasta - Chile

\title{
MESURES DE TRANSCENDANCE ET D'INDÉPENDANCE ALGÉBRIQUE DE FRACTIONS CONTINUES
}

\author{
ALI KACHA \\ Université Moulay Ismail, Marruecos
}

\begin{abstract}
Dans cet article, on donne des conditions suffisantes sur les fractions continues $A$ et $B$ pour que les nombres réels $A, B, A+B, A-$ $B, A B$ et $A / B$ soient transcendants. La méthode utilisée permet aussi de calculer une mesure de transcendance ainsi qu'une mesure d'indépendance algébrique des fractions continues transcendantes.
\end{abstract}

Classification AMS. 11A55, $11 Y 55,11 J 70$.

Mots clés. Fraction continue, mesure, transcendance, indépendance algébrique. 


\section{Introduction.}

Liouville a été le premier à démontrer l'existence de nombres transcendants. Lidée de sa méthode est qu'un nombre algébrique réel ne peut pas être " trop bien approché " par des nombres rationnels. on peut quantifier cet énoncé en termes précis mais disons simplement, à titre d'exemple, qu'on en déduit que le nombre $\sum_{n=1}^{\infty} 10^{-n !}$ est transcendant à cause de la convergence très rapide des sommes partielles de cette série. De même, on peut montrer qu'un nombre réel est transcendant si les quotients partiels de son développement en fraction continue croissent très rapidement. On peut aussi substituer au critère original de Liouville un résultat plus récent dû à Roth. Enfin, on peut généraliser la méthode pour construire des familles de nombres algébriquement indépendants.

Dans le présent travail, nous proposons la construction de familles de nombres réels transcendants en appliquant le théorème de Roth. Nous donnons par la suite une mesure de transcendance en appliquant un théorème d'A. Baker concernant l'approximation des nombres transcendants par des nombres algébriques d'un corps de nombres fixé et nous exprimons une mesure d'indépendance algébrique de ce genre de famille de nombres réels.

\section{Notations.}

Soit $\left(a_{n}\right)_{n \geq 0}$ une suite d'entiers relatifs telle que $a_{i} \geq 1$ pour tout $i \geq 1$. On note par $A=\left[a_{0}, a_{1}, a_{2}, \ldots\right]$ le fraction continue associée.

On définit les suites $\left(p_{n}\right)_{n \geq-2}$ et $\left(q_{n}\right)_{n \geq-2}$ par.

$$
\left\{\begin{array} { l l } 
{ p _ { - 2 } = 0 } & { p _ { - 1 } = 1 } \\
{ q _ { - 2 } = 1 } & { q _ { - 1 } = 0 }
\end{array} \quad \text { et } \left\{\begin{array}{l}
p_{n}=a_{n} p_{n-1}+p_{n-2} \\
q_{n}=a_{n} q_{n-1}+q_{n-2}
\end{array}, \text { pour } n \geq 0\right.\right.
$$

On pose $A_{n}=\left[a_{0}, a_{1}, a_{2}, \ldots, a_{n}\right]=\frac{p_{n}}{q_{n}}$ pour $n \geq 0$.

Rappelons les inégalités classiques (voir [3])

$$
\left|A-A_{n}\right|<\frac{1}{q_{n} q_{n+1}}<\frac{1}{a_{n+1} q_{n}^{2}},
$$

sur lesquelles on s'appuiera par la suite.

Nous tenons ausssi à rappeler un résultat de $\mathrm{A}$. Baker concernant la classification des nombres transcendants ainsi que leur mesure de transcendance que nous utiliserons par la suite. 
Théorème de A.Baker. On suppose que $\xi$ est un nombre réel ou complexe et $\tau>2$. Soient $\alpha_{1}, \alpha_{2}, \cdots$ une suite de nombres distincts d'un corps de nombres algébrique $K$ dont les hauteurs sont au plus $h\left(\alpha_{1}\right), h\left(\alpha_{2}\right), \cdots$ telles que pour tout $j \geq 1$

$$
\left|\xi-\alpha_{j}\right|<\left(h\left(\alpha_{j}\right)\right)^{-\tau} \text { et que } \lim _{j \rightarrow+\infty} \sup \frac{\log h\left(\alpha_{j+1}\right)}{\log h\left(\alpha_{j}\right)}<+\infty .
$$

soient vérifiées. Alors il existe une constante positive $\mu$ telle que

$$
\left|x_{0}+x_{1} \xi+\cdots+x_{n} \xi^{n}\right|>X^{-\mu_{n}}
$$

pour tout entier positif $n$ et toute suite d'entiers $x_{0}, x_{1}, \cdots, x_{n}$, non tous nuls, où

$$
X=\max \left(2,\left|x_{0}\right|,\left|x_{1}\right|, \cdots,\left|x_{n}\right|\right),
$$

et $\mu_{n}$ est donnée par $\log \log \mu_{n}=\mu n^{2}$.

\section{Preuve : voir [1].}

L'objet du paragraphe 2 est de donner des conditions suffisantes portant sur les quotients partiels $a_{n}$ et $b_{n}$ pour que les fractions continues $A, B, A+$ $B, A-B, A B$ et $A / B$ soient transcendantes.

Le but du troisième paragraphe est de donner une mesure de transcendance de la fraction continue $A$ en utilisant le théorème de A. Baker ci-dessus ainsi que de donner une mesure d'indépendance algébrique des deux fractions continues $A$ et $B$.

\section{Transcendance}

Soit $\left(b_{n}\right)_{n \geq 0}$ une autre suite d'entiers relatifs telle que $b_{i} \geq 1$ pour tout $i \geq 1$ dont la fraction continue associée est notée par $B=\left[b_{0}, b_{1}, b_{2}, \ldots\right]$.

Dans ce paragraphe, on pose pour tout $n \geq 0, \frac{{ }^{a} p_{n}}{{ }^{a} q_{n}}=A_{n}=$ $\left[a_{0}, a_{1}, a_{2}, \ldots, a_{n}\right]$ et $B_{n}=\frac{{ }^{a} p_{n}}{{ }^{a} q_{n}}=B_{n}=\left[b_{0}, b_{1}, b_{2}, \ldots, b_{n}\right]$.

Le principal résultat de transcendance annoncé plus haut est le suivant. 


\section{Théorème 1.}

1) S'il existe une constante réelle $\alpha>1$ et un entier $n_{0} \geq 1$ tels que pour tout $n \geq n_{0}, a_{n}>a_{n-1}^{\alpha}$ alors $A$ est transcendant.

2) S'il existe une constante réelle $\alpha>5$ et un entier $n_{1} \geq 1$ tels que pour tout $n \geq n_{1}, a_{n}>b_{n}>a_{n-1}^{\alpha}$, alors les nombres $A, B, A+B, A-B, A B$ et $A / B$ sont transcendants.

\section{Remarques.}

a) La transcendance de $A+B, A-B, A B$ et $A / B$ a étée déja démontrée par Okano [7] sous l'hypothèse plus contraignante $\alpha>\beta(n-1)$ où $\beta$ est une constante $>16$, améliorant déja un résultat de Nettler [6].

b) L'amélioration tient en particulier au fait que nous n'utilisons plus les plus formules explicites de Nettler [6].

La preuve du théorèeme 1 s'appuie sur quelques lemmes qui expriment le lien entre les quotients partiels des réels cités au théorème 1 et les dénominateurs de leurs réduites.

Lemme 1. Voir [5]. Soient $\alpha$ un nombre réel $>1$ et $n_{1}$ un entier $\geq 1$. Alors il existe une constante $C_{1}=C_{1}\left(\alpha, n_{1}\right)$ ayant la propriété suivante : Si $a_{n}>a_{n-1}^{\alpha}$ pour tout $n \geq n_{1}$, alors ${ }^{a} q_{n}<C_{1} a_{n}^{\frac{\alpha}{\alpha-1}}$.

On définit ${ }^{c} p_{n}={ }^{a} p_{n}{ }^{b} q_{n}+{ }^{b} p_{n}{ }^{a} q_{n}$ et ${ }^{c} q_{n}={ }^{a} q_{n}{ }^{b} q_{n}$ de sorte que $A_{n}+$ $B_{n}={ }^{c} p_{n} /{ }^{c} p_{n}$.

Lemme 2. S'il existe un entier $n_{2} \geq 1$ tels que $a_{n}>b_{n}$ pour tout $n \geq n_{2}$, alors ${ }^{c} q_{n}<{ }^{a} q_{n}^{2}$ pour $n$ assez grand.

Preuve : D'après la définition de $\frac{{ }^{c} p_{n}}{{ }^{c} q_{n}}$, on a ${ }^{c} q_{n} \leq{ }^{a} q_{n}{ }^{b} q_{n}<{ }^{a} q_{n}{ }^{2}$ pour tout $n$ assez grand.

Remarque : Le résultat du lemme 2 reste valable à une constante près qui dépend de $A, n_{2}$ et de $B$ lorsque ${ }^{c} p_{n}$ et ${ }^{c} q_{n}$ sont respectivement un numérateur et un dénominateur de $A_{n}-B_{n}, A_{n} B_{n}$ ou $A_{n} / B_{n}$. 


\section{Preuve du théorème 1 :}

1. Démontrons, par exemple, la transcendance de $A$. On a

$$
\left|A-\frac{{ }^{a} p_{n}}{{ }^{a} q_{n}}\right|<\frac{1}{{ }^{a} q_{n}{ }^{a} q_{n+1}}<\frac{1}{a_{n+1}{ }^{a} q_{n}^{2}} .
$$

Puisque $a_{n+1}>a_{n}^{\alpha}$ pour tout $n \geq n_{0}$ il existe, par le lemme 1 , une constante positive $C_{2}=C_{2}\left(\alpha, n_{2}\right)$ telle que $a_{n+1}>C_{2}{ }^{a} q_{n}^{\alpha-1}$. L'inégalité (2.1) ci-dessus devient:

$$
\left|A-\frac{{ }^{a} p_{n}}{{ }^{a} q_{n}}\right|<\frac{C_{2}}{{ }^{a} q_{n}^{\alpha+1}} .
$$

Comme $\alpha+1>2$, le théorème de Roth [8] nous permet de conclure.

2. (i) Montrons la transcendance de $A+B$. La suite

$$
\left(\frac{{ }^{c} p_{n}}{{ }^{c} q_{n}}\right)=\left(\frac{{ }^{a} p_{n}}{{ }^{a} q_{n}}+\frac{{ }^{b} p_{n}}{{ }^{b} q_{n}}\right)
$$

n'est pas stationnaire. En effet, pour $n$ pair, par exemple, on a

$$
A+B-\frac{{ }^{c} p_{n}}{{ }^{c} q_{n}}>0 .
$$

Par ailleurs, pour $n$ assez grand, on a

$$
\begin{gathered}
\left|A+B-\frac{{ }^{c} p_{n}}{{ }^{c} q_{n}}\right| \leq\left|A-\frac{{ }^{a} p_{n}}{{ }^{a} q_{n}}\right|+\left|B-\frac{{ }^{b} p_{n}}{{ }^{b} q_{n}}\right|<\frac{1}{{ }^{a} q_{n}{ }^{a} q_{n+1}}+\frac{1}{{ }^{b} q_{n}{ }^{b} q_{n+1}} \\
<\frac{2}{{ }^{b} q_{n}{ }^{b} q_{n+1}}<\frac{1}{b_{n+1}}<\frac{1}{a_{n}^{\alpha}} .
\end{gathered}
$$

En utilisant les lemmes 1 et 2, on obtient :

$$
\left|A+B-\frac{{ }^{c} p_{n}}{{ }^{c} q_{n}}\right|<\frac{C_{2}}{{ }^{a} q_{n}^{\alpha-1}}<\frac{C_{2}}{{ }^{c} q_{n}^{(\alpha-1) / 2}},
$$

Puisque $\alpha>5$, le théorème de Roth nous donne la transcendance de $A+B$.

(ii) Montrons que le produit $A B$ est transcendant. 
A nouveau la suite

$$
\left(\frac{{ }^{c} p_{n}}{{ }^{c} q_{n}}\right)=\left(\frac{{ }^{a} p_{n}}{{ }^{a} q_{n}} \frac{{ }^{b} p_{n}}{{ }^{b} q_{n}}\right)
$$

n'est pas stationnaire. On a

$$
\begin{gathered}
\frac{{ }^{a} p_{n}}{{ }^{a} q_{n}} \frac{{ }^{b} p_{n}}{{ }^{b} q_{n}}-\frac{{ }^{a} p_{n-1}}{{ }^{a} q_{n-1}} \frac{{ }^{b} p_{n-1}}{{ }^{b} q_{n-1}}=\left(\frac{{ }^{a} p_{n}}{{ }^{a} q_{n}}-A\right) \frac{{ }^{b} p_{n}}{{ }^{b} q_{n}}+\left(A-\frac{{ }^{a} p_{n-1}}{{ }^{a} q_{n-1}}\right) \frac{{ }^{b} p_{n}}{{ }^{b} q_{n}} \\
+\left(\frac{{ }^{b} p_{n}}{{ }^{b} q_{n}}-\frac{{ }^{b} p_{n-1}}{{ }^{b} q_{n-1}}\right) \frac{{ }^{a} p_{n-1}}{{ }^{a} q_{n-1}} \\
=(-1)^{n-1}\left(\frac{1}{\left(\frac{{ }^{a} q_{n}\left({ }^{a} q_{n}{ }^{a} \xi_{n+1}+{ }^{a} q_{n-1}\right)}{{ }^{b} p_{n}}+\frac{1}{{ }^{b} q_{n}}+\frac{1}{{ }^{a} q_{n-1}\left({ }^{a} q_{n-1}{ }^{a} \xi_{n}+{ }^{a} q_{n-2}\right)} \frac{{ }^{b} p_{n}}{{ }^{b} q_{n}}\right.}\right) \\
+(-1)^{n-1}\left(\frac{1}{{ }^{b} q_{n}{ }^{b} q_{n-1}} \frac{{ }^{a} p_{n-1}}{{ }^{a} q_{n-1}}\right)
\end{gathered}
$$

où ${ }^{a} \xi_{n}=\left[0 ; a_{n}, a_{n+1}, a_{n+2}, \ldots\right]$ d'où la conclusion, vu que les termes qui sont entre parenthèses sont tous strictement positifs.

Par ailleurs

$$
\left|A B-\frac{{ }^{c} p_{n}}{{ }^{c} q_{n}}\right| \leq\left|A-A_{n}\right||B|+\left|B-B_{n}\right|\left|A_{n}\right|<\frac{|B|+2\left|a_{0}\right|}{{ }^{b} q_{n}{ }^{b} q_{n+1}},
$$

car $\left|\frac{{ }^{a} p_{n}}{{ }^{a} q_{n}}\right| \leq 2\left|a_{0}\right|$ pour tout $n \geq 1$. Alors, il existe une constante $C_{c}$ qui dépend de $a_{0}$ et $B$ telle que

$$
\left|A B-\frac{{ }^{c} p_{n}}{{ }^{c} q_{n}}\right|<\frac{1}{b_{n+1}}<\frac{C_{2}}{{ }^{{ } q_{n}{ }^{\frac{\alpha-1}{2}}}}
$$

pour $n$ assez grand, d'où le résultat.

(iii) la démonstration est similaire pour $A-B$ et $A / B$.

\section{Exemple.}

On prend $a_{n}=2^{n !}$ pour tout $n \geq 0$ et $b_{n}=2^{6(n-1) !}$ pour $n \geq 1$ avec $b_{0}=1$. On vérifie que $a_{n}>b_{n}>a_{n-1}^{6}$ pour tout $n \geq 6$. Alors d'après le théorème 1 , les nombres réels $A, B, A+B, A-B, A B$ et $A / B$ sont transcendants. Remarquons que les résultats de [6] et de [7] ne permettent pas de conclure. 


\section{3 - Mesures de transcendance et d'indépendance algébrique.}

\subsection{Mesure de transcendance}

On gardera les notations du paragraphe 2. Pour simplifier, on notera $p_{n}={ }^{a} p_{n}$ et $q_{n}={ }^{a} q_{n}$.

Dans ce sous-paragraphe, nous donnons une mesure de transcendance de la fraction continue $A$ en utilisant le théorème de $\mathrm{A}$. Baker exprimé dans le paragraphe 1) ci-dessus.

On supose que la suite des quotients partiels $\left(a_{n}\right)$ de $A$ vérifie la chose suivante : il existe une constante réelle $\epsilon$ avec $0<\epsilon<1$, tels que,

$$
a_{n+1}=a_{n}^{1+\epsilon}, \text { pour tout } n \geq 1 \text { avec } a_{1} \geq 2,
$$

D'après le théorème 1 du paragraphe 1 , le nombre réel $A$ est transcendant.

En notant $\mathbf{Q}$ le corps des nombres rationnels, la hauteur de la réduite $A_{n+1}$ clans $\mathbf{Q}$ est est $h\left(A_{n+1}\right)=\max \left(\left|p_{n+1}\right| \cdot\left|q_{n+1}\right|\right)$. Comme $\left|A_{n+1}\right| \leq$ $(|A|+1)$, alors il existe une constante positive $C_{3}$, telle que $h\left(A_{n+1}\right) \leq$ $C_{3}\left|q_{n+1}\right|$.

De plus, par définition de la hauteur, on a $h\left(A_{n}\right) \geq q_{n}$, en utilisant le résultat du lemme 1 et les hypothèses de (2.2), on aura

$$
q_{n+1} \leq C(\epsilon) a_{n+1}^{1+\frac{1}{\epsilon}}=C(\epsilon) a_{n}^{2+\epsilon+\frac{1}{\epsilon}} .
$$

On déduit de ce qui précéde que

$$
\begin{gathered}
\frac{\log h\left(A_{n+1}\right)}{\log h\left(A_{n}\right)} \leq \frac{\log C(\epsilon)+\left(2+\epsilon+\frac{1}{\epsilon}\right) \log a_{n}}{\left(1+\frac{1}{\epsilon}\right) \log a_{n}} \\
\leq \frac{\log C(\epsilon)+\left(2+\epsilon+\frac{1}{\epsilon}\right)(1+\epsilon)^{n} \log a_{1}}{\left(1+\frac{1}{\epsilon}\right)(1+\epsilon)^{n} \log a_{1}}
\end{gathered}
$$

Il résulte de (2.3) que

$$
\lim _{n \rightarrow+\infty} \frac{\log h\left(A_{n+1}\right)}{\log h\left(A_{n}\right)} \leq \frac{2+\epsilon+\frac{1}{\epsilon}}{1+\frac{1}{\epsilon}}<+\infty .
$$

La relation (2.4) implique que le nombre réel $A$ n'est pas un U-nombre selon la classification de Mahler.

Théorème 2. Soientt $\epsilon$ une constante réelle, $0<\epsilon<1, H$ et d deux entiers positifs tels que, pour tout $n \geq 1, a_{n+1}=a_{n}{ }^{1+\epsilon}$ avec $a_{1} \geq 2$. Alors 
il existe une constante positive $\mu$ telle que pour tout polynôme $P \in Z[X]$ non nul de degré $d$ et de hauteur au plus $H$ vérifiant

$$
|P(A)|>(\max (2, H))^{-\mu_{d}}
$$

où $\mu_{d}$ est donnée par $\log \log \mu_{d}=\mu d^{2}$ et $\mu_{d}$ est indépendante de $H$.

Preuve : On sait qu'il existe une constante $C_{4}=C(\epsilon)>0$ telle que pour tout $n \geq 1$, on a

$$
\left|A-\frac{p_{n}}{q_{n}}\right|<\frac{C_{4}}{q_{n}^{2+\epsilon}} .
$$

Les inégalités (4) et (5) nous permettent d'affirmer que les hypothèses du théorème de Baker énoncé plus haut sont vérifiées. Par l'application directe de ce théorème, on prouve notre théorème 2 .

\subsection{Mesure d'indépendance algébrique}

Le théorème suivant est une version effective du résultat démontré sur l'indépendance algébrique de $A$ et $B$ dans [4].

Théorème 3. Soient $k$ un nombre réel $>1, d$ un entier $\geq 2$ et $\left(\alpha_{n}\right)_{n \geq 1}$ une suite de nombres réels $>1$, croissante et tendant vers $+\infty$, $r$ un nombre réel $>1$ tels que pour tout $n \geq 1, \quad r^{-1} a_{n}>b_{n}>a_{n-1}^{\alpha_{n-1}}$ et $a_{n-1}^{k \alpha_{n-1}}>a_{n}$. Soit $P \in Z[X, Y]$ un polynôme non nul, vérifiant $d=$ $\operatorname{deg}_{X}(P)+\operatorname{deg}_{Y}(P) \geq 2$, de hauteur au plus $H$ où $H \geq q_{2}^{\alpha_{2}}$.

Alors il existe une constante $C_{5}=C\left(A, B, d, k, \alpha_{1}\right)>0$, telle que

$$
|P(A, B)|>\frac{C_{5}}{2}(2 H)^{-\kappa} \text { ò̀ } \kappa=2 k d\left(\frac{\alpha_{1}}{\alpha_{1}-1}\right)^{2} .
$$

Preuve : Puisque $a_{n-1}^{k \alpha_{n-1}}<a_{n}$ et que $\left(\alpha_{n}\right)_{n \geq 1}$ est croissante, on a

$$
{ }^{a} q_{n}<C\left(\alpha_{1}\right) a_{n}^{\frac{\alpha_{1}}{\alpha_{1}-1}} .
$$

La deuxième hypothèse $a_{n}<a_{n-1}^{k \alpha_{n-1}}$ nous donne

$$
{ }^{a} q_{n}<C\left(\alpha_{1}\right){ }^{a} q_{n-1}^{\frac{k \alpha_{1}}{\alpha_{1}-1} \alpha_{n-1}} .
$$


On a

$$
|P(A, B)| \geq\left|P\left(A_{n}, B_{n}\right)\right|-\left|P(A, B)-P\left(A_{n}, B_{n}\right)\right| .
$$

On a déja prouvé dans [4] que $P\left(A_{n}, B_{n}\right)$ est non nul, donc

$$
\left|P\left(A_{n}, B_{n}\right)\right| \geq{ }^{a} q_{n}^{-d_{1} b} q_{n}^{-d_{2}}>{ }^{a} q_{n}^{-d},
$$

où $d_{1}=\operatorname{deg}_{X}(P)$ et $d_{2}=\operatorname{deg}_{Y}(P)$.

De plus, il existe une constante $C_{6}=C(A, B, d) \geq 1$ telle que

$$
\left|P(A, B)-P\left(A_{n}, B_{n}\right)\right|<C_{6} H\left({ }^{b} q_{n}^{b} q_{n+1}\right)^{-1} .
$$

En utilisant les inégalités $(2.9),(2.10)$ et les hypothèses sur $\left(a_{n}\right)_{n \geq 1}$ et $\left(b_{n}\right)_{n \geq 1}$, la relation (2.8) devient

$$
|P(A, B)|>{ }^{a} q_{n}{ }^{-d}-C_{6} H\left(\frac{{ }^{a} q_{n}}{C\left(\alpha_{1}\right)}\right)^{-\frac{\alpha_{1}-1}{\alpha_{1}} \alpha_{n}} .
$$

Pour avoir

$$
|P(A, B)|>\frac{1}{2^{a} q_{n}^{d}},
$$

il suffit d'avoir

$$
\frac{1}{{ }^{a} q_{n}^{d}}-C_{6} H\left(\frac{C\left(\alpha_{1}\right)}{{ }^{a} q_{n}}\right)^{\frac{\alpha_{1}-1}{\alpha_{1}} \alpha_{n}}>\frac{1}{2^{a} q_{n}^{d}} .
$$

La relation (2.12) est équivalente à

$$
{ }^{a} q_{n}{ }^{\frac{\alpha_{1}-1}{\alpha_{1}} \alpha_{n}}>C\left(\alpha_{1}\right)^{\frac{\alpha_{1}-1}{\alpha_{1}} \alpha_{n}} a_{q_{n}}{ }^{d} .
$$

Pour que (2.13) soit satisfaite, il suffit que $n$ vérifie

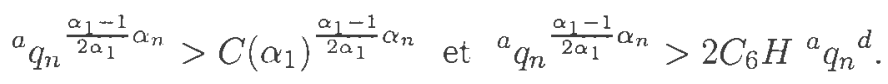

Or la première partie de l'inégalité (2.14) est facile à réaliser. Alors que sa deuxième partie est équivalente à

$$
{ }^{a} q_{n} \frac{\alpha_{1}-1}{2 \alpha_{1}} \alpha_{n}-d>2 C_{6} H .
$$

Soit $n_{1}$ le plus petit entier $\geq 2$ qui vérifie

$$
\frac{1}{2}\left(\frac{{ }^{a} q_{n-1}}{C\left(\alpha_{1}\right)}\right)^{\frac{\alpha_{1}-1}{2 \alpha_{1}} \alpha_{n-1}-d}<2 C_{6} H<\frac{1}{2}\left(\frac{{ }^{a} q_{n}}{C\left(\alpha_{1}\right)}\right)^{\frac{\alpha_{1}-1}{2 \alpha_{1}} \alpha_{n}-d} .
$$

L'entier $n_{1}$ existe car la suite $\left(\alpha_{n}\right)_{n \geq 1}$ est croissante, tend vers $+\infty$ et $H \geq \geq^{a} q_{2}^{\alpha_{2}}$. 
En utilisant (2.7) et la partie gauche de (2.15), l'inégalité (2.11) devient

$$
|P(A, B)|>\frac{1}{2 C\left(\alpha_{1}\right)^{d}}\left(2 C_{6} H\right)^{-2 k d\left(\frac{\alpha_{1}}{\alpha_{1}-1}\right)^{2}\left(1+\frac{d}{\frac{\alpha_{1}-1}{2 \alpha_{1}} \alpha_{n_{1}-1}-d}\right)} .
$$

Puisque $\frac{\alpha_{1}-1}{2 \alpha_{1}} \alpha_{n_{1}-1}-d>1,{ }^{a} q_{n_{1}-1} \geq 2$ alors en utilisant encore la partie gauche de la relation (2.15) on obtient

$$
2^{\frac{1}{\frac{\alpha_{1}-1}{2 \alpha_{1}} \alpha_{n_{1}-1}-d}}<2^{\frac{\alpha_{1}-1}{2 \alpha_{1}} \alpha_{n_{1}-1}-d} \leq 2 C_{6} H
$$

qui devient

$$
1 /\left(\frac{\alpha_{1}-1}{2 \alpha_{1}} \alpha_{n_{1}-1}-d\right)<\frac{\log \left(2 C_{6} H\right)}{\log 2} .
$$

En introduisant (2.17) dans (2.16) on aura

$$
|P(A, B)|>\frac{1}{2 C\left(\alpha_{1}\right)^{d}} \frac{1}{\left(2 C_{6} H\right)^{2 k d\left(\frac{1}{\alpha_{1}-1}\right)^{2}\left(1+\frac{d \log 2}{\log \left(2 C_{6} H\right)}\right)}} .
$$

Ce qui prouve le résultat.

Exemple. Soient $a_{n}=2^{\frac{(2 n) !}{2^{n+1}}}$ et $b_{n}=2^{\frac{(2 n-1) !}{2^{n}}}$ pour tout $n \geq 3$, avec $a_{0}=b_{0}=0, a_{1}=b_{1}=1, a_{2}=8$ et $b_{2}=2$. Soit $P$ un polynôme quadratique de hauteur $H \geq 81$, alors il existe une constante positive $C_{7}=C(A, B)$ telle que

$$
|P(A, B)|>\frac{C_{7}}{2}(4 H)^{-281} .
$$




\section{Références}

1. A. BAKER - On Mahler's classification of transcendental numbers, Acta Matematica 111, pp. 97-120, (1964)

2. P. BUNDSCHUH - Transcendental continued fractions, J. Number Theory, 18, pp. 91-98, (1984).

3. G.H. HARDY and E.M. WRIGHT - An introduction to the Theoryof Numbers, Oxford Univ. Press, (1985).

4. A. KACHA - Approximation algébrique de fractions continues, C.R. Acad. Sci. Paris, t 317, Série 1, pp. 17-20, (1993).

5. A. KACHA - Transcendance et fractions continues, Séminaire de Théorie des Nombres 1990, Université de Caen.

6. G. NETTLER - Transcendental continued fractions, J. Number Theory, 13, pp. 456-462, (1981).

7. T. OKANO - A note on the transcendental continued fractions; Tokyo J. Math Vol. 10, n1, pp. 152-156, (1987).

8. A.B. SHIDLOVSKII - Transcendental numbers, Walter de Gruyter (1989).

Received : April, 1999.
Ali Kacha
Université Moulay Ismail
Faculté des Sciences et Techniques
Département de Mathématiques
B.P 509, Boutalamine
Errachidia - MAROC 